\title{
Portfolio Optimization in Jump Model under Inefficiencies in the Market
}

\author{
Dereje Bekele ${ }^{1 *}$, Ananda Kube², Dennis C. Ikpe ${ }^{3}$ \\ ${ }^{1}$ Pan African University Institute for Basic Sciences, Technology and Innovation (PAUISTI), Juja, Kenya \\ ${ }^{2}$ Department of Statistics and Actuarial Sciences, Kenyatta University, Nairobi, Kenya \\ ${ }^{3}$ Department of Mathematical Sciences, University of South Africa, Florida, South Africa \\ Email: *derebekele34@gmail.com
}

How to cite this paper: Bekele, D., Kube, A. and Ikpe, D.C. (2018) Portfolio Optimization in Jump Model under Inefficiencies in the Market. Journal of Mathematical Finance, 8, 562-575.

https://doi.org/10.4236/jmf.2018.83036

Received: April 1, 2018

Accepted: August 6, 2018

Published: August 9, 2018

Copyright ( $) 2018$ by authors and Scientific Research Publishing Inc. This work is licensed under the Creative Commons Attribution International License (CC BY 4.0). http://creativecommons.org/licenses/by/4.0/

\begin{abstract}
This paper evaluates the use of modeling approach that depends on Levy jump model to predict investors wealth under inefficiencies in the market, in terms of mispricing and asymmetric information where the traded stock or risky asset price is considered to be as a function of a Levy jump process (i.e. the driving Levy process has Brownian component) by specifying the asset price process in the large filtration of informed investor. Then we obtain its dynamics for uninformed investor using the Hitsuda representation of Gaussian processes assuming there are two distinct classes of rational investors. In this setting assuming power utility functions, the optimal portfolios, maximum expected power utilities and asymptotic utilities for investors from the terminal wealth are derived by the methods of optimization and stochastic calculus.
\end{abstract}

\section{Keywords}

Information Asymmetry, Inefficiencies, Optimal Portfolio, Jump Model, Power Utilities

\section{Introduction}

Nowadays, the essential topic in the financial engineering is the selection of the portfolios and asset pricing. If the market is efficient, then it is expected that asset prices reveal the existing information, and all investors have the same amount of information to select portfolios. However, one of the most remarkable developments of the last few decades was the most extremely thought concepts of market efficiency, the positive relationship among return and non-diversifiable risk. This was due to the strong unexpected price volatility 
in the markets such as stock, bond, currency and real estate markets.

[1] and [2] were among the first to emphasize that there are many market anomalies including excess volatility caused by investor overreaction and under-reaction, fashions and mispricing on their empirical studies thought on this area. [3], [4] and [5] are among current behavioral finance articles discussed for the presence of these market anomalies. Information asymmetry has a considerable outcome on asset prices and difficulties which upsets assets are through a liquidity channel [3]. Asset mispricing and information asymmetry connection in a purely deterministic and discrete setting was first studied by [5] and [6] and later prolonged to the purely continuous random environment by [7] and [8]. So that, using this as a background asset pricing and portfolio selection should be studied in an inefficient framework by assuming that the asset has both the fundamental value and market value and there are two types of investors in the market as informed who observes both fundamental and market value and also has non-public information advantage to trade and uninformed investor observes market values and makes investment choices using public information only.

Levy processes have gained extensive interest in financial modeling as they were found to overcome many of the shortcomings associated with the Black-Scholes model and to offer a more general tool for modeling inefficiency in asset prices. The probability distributions associated with Levy processes are infinitely divisible and offer more flexibility for fitting financial data. Mispricing models for stocks under asymmetric information were first studied by [6] in a purely deterministic setting and [7] extends to the continuous random environment where he has assumed that stock prices follow geometric Brownian motion, utility function from logarithmic and the continuous mean-reverting Ornstein-Uhlenbeck $(\mathrm{O}-\mathrm{U})$ process represents the mispricing in the market. Moreover, he has derived optimal portfolios and maximum expected logarithmic utilities, including asymptotic utilities for both rational investors (uninformed and informed).

In this paper, we followed the same modeling approach using Levy jump model to predict investors wealth under inefficiencies in the market, in terms of mispricing and asymmetric information by specifying the asset price process in the large filtration of informed investor. Then we obtain its dynamics for uninformed investor using the Hitsuda representation of Gaussian processes assuming there are two distinct classes of rational investors. In this setting assuming power utility functions, the optimal portfolios, maximum expected power utilities and asymptotic utilities for investors from the terminal wealth are derived by the methods of optimization and stochastic calculus.

\section{The Model}

The model consists of two assets a risk-less asset $B$ called bond earns a continuously compounded risk-free interest rate $r_{t}$ and a risky asset $S$ on a 
probability space $(\Omega, \mathcal{F}, P)$ called stock with with total percentage appreciation rate or expected returns $\mu_{t}$ at time $t \in[0, T]$ given as follows respectively.

$$
\begin{gathered}
B_{t}=\exp \left(\int_{0}^{t} r_{s} \mathrm{~d} s\right) \\
d\left(\log S_{t}\right)=\left(\mu_{t}-\frac{1}{2} \sigma_{t}^{2}\right) \mathrm{d} t+\sigma_{t} \mathrm{~d} X_{t}+\mathrm{d} Z_{t}, \quad \text { where } \mathrm{d} Z_{t}=\int_{R} z N(\mathrm{~d} t, \mathrm{~d} z)
\end{gathered}
$$

The market parameters are $\mu_{t}, \sigma_{t}$ and $r_{t}$ are Lebesgue integrable deterministic functions.

$X$ is defined by,

$$
X_{t}=p W_{t}+q U_{t}, p^{2}+q^{2}=1, p \geq 0, q \geq 0,
$$

to embody both a permanent component and a temporary component of price shocks represented by $W_{t}$ and $U_{t}$ respectively. The mean-reverting O-U process $U=\left(U_{t}\right)_{t \geq 0}$ with rate $\lambda$ representing the mis pricing are defined exactly as [7] and satisfies the Langevin stochastic differential equation given by:

$$
\mathrm{d} U_{t}=-\lambda U_{t} \mathrm{~d} t+\mathrm{d} B_{t}, \lambda \geq 0, U_{0}=0,
$$

with a unique solution $U_{t}=U_{0} \mathrm{e}^{-\lambda t}+\int_{0}^{t} \mathrm{e}^{-\lambda(t-s)} \mathrm{d} B s=\int_{0}^{t} \mathrm{e}^{-\lambda(t-s)} \mathrm{d} B s$ with

$E\left[U_{t}\right]=0$ and $E\left[U_{t}^{2}\right]=\operatorname{Var}\left(U_{t}\right)=\frac{1}{2 \lambda}\left(1-\mathrm{e}^{-2 \lambda t}\right)$.

The stock's Sharpe ratio or market price of risk $\theta$ is square integrable $T>0$ is the investment horizon. $Z$ is a pure jump Levy process having a $\sigma$-finite Levy measure $v$ on $\mathcal{B}(R-\{0\})$ with triple $(\alpha, 0, v)$ where $\alpha=\int_{|z| \leq 1} z v(\mathrm{~d} z)$ and the Levy measure satisfies $v(\{0\})=0, \int_{R}\left(1 \vee z^{2}\right) v(\mathrm{~d} z)<\infty \quad N$ is a Poisson random measure on $R_{+} \times \mathcal{B}(R-\{0\})$ that is linked to the stock. It counts the jumps of $Z$ in the time interval $(0, t)$.

The returns of the stock has three components; a continuous component $\mu_{t}^{*}=\mu_{t}-\frac{1}{2} \sigma_{t}^{2}$, a diffusive component $\sigma_{t} X_{t}$ which is random, and a discontinuous component $\mathrm{d} Z_{t}$, which is also random.

The continuous component of the stock's return $\mu_{t}^{*}$ and the volatility $\sigma_{t}$, are assumed to be deterministic functions with $\sigma=\lim _{t \rightarrow \infty} \sigma_{t}=\sigma_{\infty}>0$.

The process $X=\left(X_{t}\right)_{t \geq 0}$, the continuous random component of excess return and the over all process is assumed with finite mean and variance. By the Levy-Itô decomposition theorem [9] $Z$ is written as $Z_{t}=\alpha t+\int_{|z|<1} z \tilde{N}(t, \mathrm{~d} z)+\int_{|z| \geq 1} z N(t, \mathrm{~d} z)$, with triplet $(\alpha, 0, v)$ and $\alpha=\int_{|z|<1} z v(\mathrm{~d} z)$ and $\mathrm{d} Z_{t}=\int_{R} z N(\mathrm{~d} t, \mathrm{~d} z)$ then the log return dynamic becomes:

$$
\mathrm{d}\left(\log S_{t}\right)=\left(\mu_{t}-\frac{1}{2} \sigma_{t}^{2}\right) \mathrm{d} t+\sigma_{t} \mathrm{~d} X_{t}+\int_{R} z N(\mathrm{~d} t, \mathrm{~d} z), t \in[0, T]
$$

And by using Itô's formula to Equation (5) yields percentage returns:

$$
\frac{\mathrm{d} S_{t}}{S_{t^{-}}}=\mu_{t} \mathrm{~d} t+\sigma_{t} \mathrm{~d} X_{t}+\int_{R}\left(\mathrm{e}^{z}-1\right) N(\mathrm{~d} t, \mathrm{~d} z), t \in[0, T] .
$$


All the investors random objects are defined on the filtered probability space $\left(\Omega, \mathcal{F}, \mathcal{H}^{j}, P\right) j=\{0,1\}$. We used index 1 and 0 for informed and uninformed investors respectively. The information flows of the informed and uninformed investors is written as follows:

$$
\mathcal{H}_{t}^{1}=\sigma\left(W_{s}, B_{s}, Z_{s}: s \leq t\right) \vee \sigma(\mathcal{N}), \mathcal{H}_{t}^{0}=\mathcal{F}_{t}^{0} \vee \sigma\left(Z_{l}: l \leq t\right), t \in[0, T]
$$

where $\mathcal{F}_{t}^{0}=\sigma\left(X_{s}: s \leq t\right)$ is given by [7]. $Z$ and $X$ are independent, since $Z, W, U$ are independent with $\mathcal{F}_{t}^{0} \subset \mathcal{H}_{t}^{0} \subset \mathcal{H}_{t}^{1} \subset \mathcal{F}$

\section{Dynamics of Asset Price}

\subsection{Dynamics of Asset Price for Informed Investor}

Based on information flow of informed investor from Equations (3) and (5) $\mathrm{d} X_{t}=v_{t}^{1} \mathrm{~d} t+\mathrm{d} B_{t}^{i}$ where $v_{t}^{1}=-\lambda q U_{t}$ and $B_{t}^{1}=p W+q B$, then the percentage return dynamics of informed investor is,

$$
\frac{\mathrm{d} S_{t}}{S_{t^{-}}}=\mu_{t}^{1} \mathrm{~d} t+\sigma_{t} \mathrm{~d} B_{t}^{1}+\int_{R}\left(\mathrm{e}^{z}-1\right) N(\mathrm{~d} t, \mathrm{~d} z), \quad \text { where } \mu^{1}=\mu_{t}+v_{t}^{1} \sigma_{t} .
$$

By using Levy characterization theorem [10] the price process is,

$$
S_{t}=S_{0} \exp \left(\int_{0}^{t} \mu_{s}^{1} \mathrm{~d} s-\frac{1}{2} \int_{0}^{t} \sigma_{s}^{2} \mathrm{~d} s+\int_{0}^{t} \sigma_{s} \mathrm{~d} B_{s}^{1}+\int_{R} z N(\mathrm{~d} t, \mathrm{~d} z)\right)
$$

\subsection{Dynamics of Asset Price for Uninformed Investor}

Using the representation of Gaussian process from [11], [7] gave an $\mathcal{H}^{0}$ -Brownian motion $B^{0}$ and a random process $v^{0}$, such that $\mathrm{d} X_{t}=\mathrm{d} B_{t}^{0}+v_{t}^{0} \mathrm{~d} t$ on probability space $(\Omega, \mathcal{F}, P)$ which is independent from Brownian motion $B$ and $W$, then the percentage return dynamics of uninformed investor equating with Equation (6) is given by:

$$
\frac{\mathrm{d} S_{t}}{S_{t^{-}}}=\mu_{t}^{0} \mathrm{~d} t+\sigma_{t} \mathrm{~d} B_{t}^{0}+\int_{R}\left(\mathrm{e}^{z}-1\right) N(\mathrm{~d} t, \mathrm{~d} z), \quad \text { where } \mu_{t}^{0}=\mu_{t}+\sigma_{t} v_{t}^{0}
$$

with price

$$
S_{t}=S_{0} \exp \left(\int_{0}^{t} \mu_{s}^{0} \mathrm{~d} s-\frac{1}{2} \int_{0}^{t} \sigma_{s}^{2} \mathrm{~d} s+\int_{0}^{t} \sigma_{s} \mathrm{~d} B_{s}^{0}+\int_{R} z N(\mathrm{~d} t, \mathrm{~d} z)\right)
$$

where $v_{s}^{0}=-\lambda \int_{0}^{s}(\alpha(s)+1) \mathrm{e}^{\lambda(s-u)} \mathrm{d} B_{s}^{u}$ with $\alpha(s)=\frac{1-p^{2}}{1+p \tanh (p \lambda s)}-1$

Remark 3.3. $\alpha(u)$ is the solution of the Cauchy equation given by,

$$
\alpha^{\prime}(s)=\lambda\left(\alpha^{2}(s)-p^{2}\right), \alpha(0)=-p^{2}
$$

Lemma 3.4. Let $p \in[0,1]$ and $t \in[0, T]$. The $E\left[v^{0}\right]=0$,

$$
\begin{aligned}
& E\left[v^{0}\right]^{2}=\lambda^{2} \int_{0}^{t} \mathrm{e}^{-2 \lambda(t-s)}(1-\alpha(s))^{2} \mathrm{~d} s, \lim _{t \rightarrow \infty} E\left[v^{0}\right]^{2}=\frac{\lambda}{2}(1-p)^{2}, \\
& \int_{0}^{T} E\left[v^{0}\right]^{2}=\frac{\lambda}{2}(1-p)^{2}, \text { as } T \rightarrow \infty \text { and } \lim _{t \rightarrow \infty} E\left[v^{1}\right]^{2}=\frac{\lambda}{2}(1-p)(1+p),
\end{aligned}
$$


$\int_{0}^{T} E\left[v^{1}\right]^{2}=\frac{\lambda}{2}(1-p)(1+p)$, as $T \rightarrow \infty$

The stock percentage return dynamic for the investors relative to the filtration with $j \in\{0,1\}$.

$$
\frac{\mathrm{d} S_{t}}{S_{t^{-}}}=\mu_{t}^{j} \mathrm{~d} t+\sigma_{t} \mathrm{~d} B_{t}^{j}+\int_{R}\left(\mathrm{e}^{z}-1\right) N(\mathrm{~d} t, \mathrm{~d} z), t \in[0, T]
$$

\section{Wealth and Portfolio Dynamics of Investors}

Before going to the main results we introduce some preliminary concepts on portfolio and wealth process.

Definition 4.1. Portfolio process

A portfolio $\Pi^{j}:[0, T] \times \Omega \rightarrow R$, is an $\mathcal{H}=\mathcal{H}_{t}^{j} \geq 0$-adapted process satisfying

$$
\int_{0}^{T}\left(\Pi_{t}^{j} \sigma_{t}\right)^{2} \mathrm{~d} t<\infty \text { almost surely }
$$

where $\Pi$ is a function of $(t, \omega)$ and $\omega$ is kept as the background and it is assumed that $\Pi^{j}$ is a function of time $t$ and $\Pi_{t}^{j}$ is the proportion of an investor's wealth invested in the stock at time $t$. The remainder $1-\Pi_{t}^{j}$ is invested in the bond or money market.

Definition 4.2. Wealth process

The wealth process for the investors is $W^{j, \Pi, x}:[0, T] \times \Omega \rightarrow R$, where $W_{t}^{j, \Pi, x}$ is the value of the portfolio (stock and bond) at time $t . \Pi_{t}$ is the proportion of the wealth invested in the stock. $x>0$ is the initial capital which is $W_{0}^{j}=x$. For the sake simplicity, it will be denoted by $W^{j}=\left(W_{t}^{j, \Pi, x}\right)_{t \geq 0}$

Let $W_{t}=W_{t}^{j}$ be the wealth process of the uninformed investors at time $t$ as a result of investing $\Pi_{t}=\Pi_{t}^{j}$ in the stock.

Let $n_{t}^{j}=m_{t}$ is the number of stocks in the portfolio at the time $t$. Then

$$
\Pi_{t}=\Pi_{t}^{j}=\frac{m_{t} S_{t^{-}}}{W_{t^{-}}^{j}}
$$

where $W_{t^{-}}^{j}$ is the value of the portfolio just before time $t$. Now by assuming all portfolios are self-financing,

$$
\begin{aligned}
& \mathrm{d} W_{t}^{j}=\left(1-\Pi_{t}\right) W_{t^{-}}^{j} r_{t} \mathrm{~d} t+m_{t} \mathrm{~d} S_{t}=\mathrm{d} W_{t}^{j}=\left(1-\Pi_{t}\right) W_{t^{-}}^{j} r_{t} \mathrm{~d} t+\Pi_{t} W_{t^{-}}^{j} \frac{\mathrm{d} S_{t}}{S_{t^{-}}} \\
& \text {so, } \frac{\mathrm{d} W_{t}^{j}}{W_{t^{-}}^{j}}=\left(1-\Pi_{t}\right) r_{t} \mathrm{~d} t+\Pi_{t} \frac{\mathrm{d} S_{t}}{S_{t^{-}}}
\end{aligned}
$$

By substituting the asset return dynamics Equation (11) in (13) and using Stochastic Exponential the wealth process $W_{t}^{j}$ has dynamics.

$$
\frac{\mathrm{d} W_{t}^{j}}{W_{t^{-}}^{j}}=\left(\theta_{t}^{j} \Pi_{t} \sigma_{t}+r_{t}\right) \mathrm{d} t+\Pi_{t} \sigma_{t} \mathrm{~d} B_{t}^{j}+\Pi_{t} \int_{R}\left(\mathrm{e}^{z}-1\right) N(\mathrm{~d} t, \mathrm{~d} z)
$$

where $\theta_{t}^{j}=\frac{\mu_{t}^{j}-r_{t}}{\sigma_{t}}$ is market price of risk or Sharpe ratio.

The discounted value $\tilde{W}_{t}^{j}=W_{t}^{j} \exp \left(-\int_{0}^{t} r_{t} \mathrm{~d} s\right)$ is given by: 


$$
\begin{aligned}
\tilde{W}_{t}^{j}= & W_{t^{-}}^{j} \exp \left(\int_{0}^{t}\left(\Pi_{s} \sigma_{s} \theta_{s}^{u}-\frac{1}{2} \Pi_{s}^{2} \sigma_{s}^{2}\right) \mathrm{d} s+\int_{0}^{t} \Pi_{s} \sigma_{s} \mathrm{~d} B_{s}^{j}\right. \\
& \left.+\int_{0}^{t} \int_{R} \log \left(1+\Pi_{s}\left(\mathrm{e}^{z}-1\right) N(\mathrm{~d} s, \mathrm{~d} z)\right)\right) .
\end{aligned}
$$

Remark 4.3. The interest rate $r_{t}$ is set to be zero in the following discussion then the terminal wealth $W_{T}^{j}$ is equivalent to to the discounted terminal wealth $\tilde{W}_{t}^{j}$.

\section{Optimal Portfolio and Power Utility Maximization}

It is assumed that each investor has a utility function $U:(0, \infty) \rightarrow R$ that satisfies the Inanda condition. A function $U:(0, \infty) \rightarrow R$ satisfies Inanda Condition, if it is strictly increasing, strictly concave, continuously differentiable with: $\frac{\mathrm{d} U}{\mathrm{~d} x}(0)=\lim _{x \rightarrow 0} \frac{\mathrm{d} U}{\mathrm{~d} x}(x)=+\infty, \frac{\mathrm{d} U}{\mathrm{~d} x}(\infty)=\lim _{x \rightarrow 0} \frac{\mathrm{d} U}{\mathrm{~d} x}(x)=0$. We assumed power utility function which is defined by $U_{\alpha}(x)=\frac{x^{\alpha}}{\alpha}, \alpha<1$ with constant relative risk aversion (RRA), $1-\alpha$.

Particularly it shown that $U_{\alpha}(x)=\frac{x^{\alpha}-1}{\alpha}, \rightarrow U_{0}(x)=\log (x)$, when $\alpha \rightarrow 0$, $U(x)=x^{\alpha}=\mathrm{e}^{\alpha \log x}$.

By defining

$$
G^{j}(t)=\int_{0}^{t}\left(\Pi_{s} \sigma_{s} \theta_{s}^{j}-\frac{1}{2} \Pi_{s}^{2} \sigma_{s}^{2}\right) \mathrm{d} s+\int_{0}^{t} \Pi_{s} \sigma_{s} \mathrm{~d} B_{s}^{j}+\int_{0}^{t} \int_{R} \log \left(1+\Pi_{s}\left(\mathrm{e}^{z}-1\right) N(\mathrm{~d} s, \mathrm{~d} z)\right)
$$

and taking the expectation of $G^{j}(t)$ with

$$
\begin{aligned}
& H(\Pi)=\int_{R} \log \left(1+\Pi\left(\mathrm{e}^{z}-1\right)\right) v(\mathrm{~d} z) \\
E G^{j}(t)= & \frac{1}{2} E \int_{0}^{T}\left(2 \Pi_{s} \sigma_{s} \theta_{s}^{j}-\Pi_{s}^{2} \sigma_{s}^{2}\right) \mathrm{d} s+E \int_{0}^{T} \Pi_{s} \sigma_{s} \mathrm{~d} B_{s}^{j} \\
& +\alpha E \int_{0}^{T} \int_{R} \log \left(1+\Pi_{s}\left(\mathrm{e}^{z}-1\right)\right) N(\mathrm{~d} s, \mathrm{~d} z) \\
= & \frac{1}{2} E \int_{0}^{T}\left(2 \Pi_{s} \sigma_{s} \theta_{s}^{j}-\Pi_{s}^{2} \sigma_{s}^{2}\right) \mathrm{d} s+E \int_{0}^{t} H\left(\Pi_{s}^{j}\right) \mathrm{d} s
\end{aligned}
$$

and by Ito's Isometry $\operatorname{Var}\left(G^{j}(t)\right)=\int_{0}^{t}\left(\Pi_{s} \sigma_{s}\right)^{2} \mathrm{~d} s$.

The expectation of $U\left(\tilde{W}_{T}^{j, \Pi}\right)$ is given by: $E U\left(\tilde{W}_{T}^{j, \Pi}\right)=E \mathrm{e}^{\alpha \log x+\alpha G^{j}(t)}$, the following result is written for the expected power utility for investors from the terminal wealth.

Theorem 5.1. The expected power utility for investors from the terminal wealth $\tilde{W}_{t}^{j}$ is given by:

$$
U(x)=E U\left(\tilde{W}_{T}^{j, \Pi}\right)=\mathrm{e}^{\alpha \log x+\frac{\alpha}{2}(1-\alpha) E \int_{0}^{t}\left(\frac{\theta_{s}^{j}}{1-\alpha}\right)^{2} \mathrm{~d} s+\frac{\alpha}{1-\alpha} E \int_{0}^{t} j\left(\Pi_{t}^{j}\right) \mathrm{ds}},
$$

where $g^{j}\left(\Pi_{t}^{j}\right)=-\frac{1}{2}\left(\frac{\theta_{t}^{j}}{1-\alpha}-\Pi_{t} \sigma_{t}\right)^{2}+\frac{\alpha}{1-\alpha} H\left(\Pi_{t}^{j}\right), \quad \theta_{t}^{j}=\frac{\mu_{t}^{j}-r_{t}}{\sigma_{t}}$ and

$$
\mu_{t}^{j}=\mu_{t}+\sigma_{t} v_{t}^{j} .
$$


The admissible set for the investors is denoted by $C_{j}(x)$ where

$$
\begin{aligned}
C_{j} & =\left\{\Pi^{j}: W_{t}^{j, \Pi^{j}, x}>0, \Pi^{j} \text { is } \mathcal{H}^{j} \text { predictabl, } S \text {-intigrable }\right\} \\
& =\left\{\Pi^{j}: \tilde{W}_{t}^{j, \Pi^{j}, x}>0, \Pi^{u} \text { is } \mathcal{H}^{j} \text { predictabl, } S \text {-intigrable }\right\}
\end{aligned}
$$

A process $\Pi=\Pi^{j}$ is called admissible if it a member of $C_{j}(x)$. It is assumed that $\tilde{W}^{j}$ is the discounted wealth process of $W^{j}$, and $\Pi$ is predictable if it is measurable with respect to the predictable sigma-algebra of left continuous with right limit function on $[0, T] \times \Omega$.

The optimal portfolio for the investors $\Pi^{j^{*}} \in C_{j}(x)$ such that

$$
E\left[U\left(\tilde{W}_{T}^{j, \Pi^{*}}\right)\right]=\max _{\Pi \in C_{j}(x)} E\left[U\left(\tilde{W}_{T}^{j, \Pi}\right)\right]
$$

which means $\Pi^{j^{*}}=\arg \max _{\Pi \in C_{j}(x)} E\left[U\left(\tilde{W}_{T}^{j, \Pi}\right)\right]$.

From theorem (5.1) $\theta_{t}^{j}$ is independent of $\Pi_{t}$. So $E\left[\mathrm{e}^{\alpha \log W_{T}^{j}}\right]$ is maximized if and only if $E\left[\int_{0}^{T} g^{j}\left(\Pi_{s}^{j}\right) \mathrm{d} s\right]$ maximized which means, $g^{j}\left(\Pi_{t}^{j}\right)$ is maximized on the admissible set $C_{j}(x)$.

By using similar approach to the optimization method used by [12] [13] [14], and yields the same optimal as the HJB approach.

The major result as unique admissible optimal portfolio and the maximum expected power utility are presented in the following theorem.

Theorem 5.2. Assume that the first and second differentiation of H(.) with respect $\Pi$ exist.

1) Let $\Pi^{j} \in C_{j}(x)$ and

$$
\begin{aligned}
g\left(\Pi^{j}\right) & =-\Pi_{t}^{j}+\frac{\theta_{t}^{j}}{(1-\alpha) \sigma_{t}}+\frac{\alpha H^{\prime}\left(\Pi_{t}^{j}\right)}{2(1-\alpha) \sigma_{t}^{2}} \\
& =-\Pi_{t}^{j}+\frac{\theta_{t}^{j}}{(1-\alpha) \sigma_{t}}+\frac{\alpha}{2(1-\alpha) \sigma_{t}^{2}} \int \frac{\left(\mathrm{e}^{z}-1\right) v \mathrm{~d} z}{1+\Pi^{j}\left(\mathrm{e}^{z}-1\right)} \quad \text { with } j \in\{0,1\}
\end{aligned}
$$

$g^{j}\left(\Pi_{t}^{j}\right)$ is strictly concave on $R$, with unique maximum optimal portfolio $\Pi^{j^{*}}$ for each investor that satisfies $g\left(\Pi^{j^{*}}\right)=0, t \in[0,1]$. Further more, $g(0)=\frac{\theta_{t}^{j}}{(1-\alpha) \sigma}+\frac{\alpha}{2(1-\alpha) \sigma^{2}} \int\left(\mathrm{e}^{z}-1\right) v \mathrm{~d} z>0$ and $g(1)=-1+\frac{\theta_{t}^{j}}{(1-\alpha) \sigma}+\frac{\alpha}{2(1-\alpha) \sigma^{2}} \int\left(1-\mathrm{e}^{-z}\right) v \mathrm{~d} z<0$ then there is unique admissible optimal portfolio $\Pi_{t}^{j^{*}} \in C_{j}(x)$

2) For $f^{\text {th }}$ investor the maximum expected power utility from terminal wealth for investors with initial wealth $x>0$ is given by

$$
U^{j}(x)=\max _{\Pi \in C_{j}(x)} E \mathrm{e}^{U\left(\tilde{W}_{T}^{j, \Pi}\right)}=\mathrm{e}^{\alpha \log x+\frac{\alpha}{2(1-\alpha)} E \int_{0}^{T}\left(\frac{\mu_{t}+v_{t}^{j} \sigma_{t}}{\sigma_{t}}\right)^{2} \mathrm{~d} s+\frac{\alpha}{2(1-\alpha)} E \int_{0}^{T} g^{j}\left(\Pi_{t}^{j^{*}}\right) \mathrm{d} s}
$$

where $\Pi_{t}^{j^{*}}=\frac{\theta_{t}^{j}}{(1-\alpha) \sigma_{t}}+\frac{\alpha H^{\prime}\left(\Pi_{t}^{j^{*}}\right)}{2(1-\alpha) \sigma_{t}^{2}} \in C_{j}(x)$ is the optimal portfolio of 
investors.

Remark 5.1. The optimal portfolio $\Pi^{* j}$ is random and becomes deterministic when there is no information asymmetry. Since the drift term is random the dynamics of the return are random in the presence of information asymmetry in which optimal demand for the risky asset is random. If there is no information asymmetry investors perceive both fundamental and market values of the asset. Hence, there is no mispricing, which means $U=0$, and therefore the return dynamics are the same, which yielding a common deterministic continuous optimal.

We analyzed theorem (5.1) in terms of continuous and jump component of the the portfolio $\Pi_{t}$ by defining for $t \in[0, T]$ as $\Pi_{t, c}^{j}=\frac{\theta_{t}}{(1-\alpha) \sigma_{t}}$ and $\Pi_{t, J}^{j}=\frac{\alpha H^{\prime}\left(\Pi_{t}^{j}\right)}{2(1-\alpha) \sigma_{t}^{2}}$ respectively and $U_{T, c}^{j}(x)=\mathrm{e}^{\alpha \log x+\frac{\alpha}{2(1-\alpha)} E \int_{0}^{T}\left(\frac{\mu_{t}+v_{v}^{j} \sigma_{t}}{\sigma_{t}}\right)^{2} \mathrm{~d} s}$ and $U_{T, J}^{j}(x)=\mathrm{e}^{\frac{\alpha}{2(1-\alpha)} E \int_{0}^{T} g^{j}\left(\Pi_{t}^{j *}\right) \mathrm{ds}}$ is the maximum expected power utility on an optimal portfolio $\Pi$ with investment horizon $T$ which is assumed to be adapted to their filtration.Using this notation Theorem (5.1) 1) states for both investors, there is unique optimal portfolio $\Pi^{{ }^{* j}} \in C_{j}(x)$, such that $\Pi^{{ }^{*} j}=\Pi_{c}{ }^{* j}+\Pi_{J}^{{ }^{* j} j}$, where $\Pi_{c}^{{ }^{* j}}$ is the continuous optimal portfolio for an asset with Geometric Brownian motion dynamics $\mathrm{d} S_{t}=\mu_{t} S_{t} \mathrm{~d} t+\sigma_{t} S_{t} \mathrm{~d} B_{t}$ and $\Pi_{J}^{* j}$ is the excess asset holding resulting from the jumps for $f^{\text {th }}$ investor. Further more, 2) establishes that the maximum expected power utility from the terminal wealth for each investors, having $x>0$ initial wealth is given by

$u^{j}=U_{T}^{j}(x)=U_{T, c}^{j}(x)+U_{T, J}^{j}(x)$ which means, $u=U_{T}(x)=U_{T, c}(x)+U_{T, J}(x)$, where $U_{T, c}(x)$ is the maximum expected power utility from the terminal wealth for the continuous part with optimal portfolio $\Pi_{c}^{*}$ and $U_{T, J}^{j}(x)$ is the excess utility from the jump.

The value $U_{T, J}^{j}(x)$ depends on the growth rate of $H$ and optimality is achieved if $H$ is twice differentiable. That is, if $H^{\prime \prime}(\Pi)=-\int_{R} \frac{\left(\mathrm{e}^{z}-1\right) v(\mathrm{~d} z)}{\left(1+\Pi\left(\mathrm{e}^{z}-1\right)\right)^{2}}$ finitely exists. If $\Pi \in[0,1)$, then $H^{\prime \prime}(\Pi)<\infty$ when ever $\int_{R}\left(\mathrm{e}^{z}-1\right)^{2} v(\mathrm{~d} z)<\infty$. If $\Pi=1$, then $H^{\prime \prime}(\Pi)$ exists if $\int_{R}\left(\mathrm{e}^{-z}-1\right)^{2} v(\mathrm{~d} z)<\infty$. Hence, Theorem (5.1) is true if no short-selling $(\Pi<0)$ or borrowing $(\Pi>1)$ from the bank account is allowed, and $\int_{R}\left(\mathrm{e}^{ \pm}-1\right)^{2} v(\mathrm{~d} z)<\infty$.

\subsection{Holding of Excess Stock by Investors}

Define the continuous optimal for informed and uninformed investors by:

$$
\gamma \triangleq \frac{\theta_{t}}{(1-\alpha) \sigma_{t}}=\Pi_{t, c}^{* j}
$$

Let $H\left(\Pi_{t}^{j}\right)=\int_{R} \log \left(1+\Pi_{t}\left(\mathrm{e}^{z}-1\right)\right) v(\mathrm{~d} z)$ with $\Pi \in[0,1]$, 
$\int_{R}\left(\mathrm{e}^{z}-1\right)^{2} v(\mathrm{~d} z)<\infty$ then $H^{\prime \prime}(\Pi)<0$ and from Theorem (5.1) the optimal portfolio $\Pi_{t}^{*}$ of investors is:

$$
\begin{aligned}
& \Pi_{t}^{j^{*}}=\frac{\theta_{t}^{u}}{(1-\alpha) \sigma_{t}}+\frac{\alpha H^{\prime}\left(\Pi_{t}^{j^{*}}\right)}{2(1-\alpha) \sigma_{t}^{2}}=\gamma+\frac{\alpha H^{\prime}\left(\Pi_{t}^{j^{*}}\right)}{2(1-\alpha) \sigma_{t}^{2}} \\
& \Pi_{t}^{u^{*}}-\gamma=k \frac{H^{\prime}\left(\Pi_{t}^{j^{*}}\right)}{\sigma_{t}^{2}} \text { where } k=\frac{\alpha}{2(1-\alpha)} \text {, so } \\
& H^{\prime}\left(\Pi_{t}^{j^{*}}\right)=\frac{\sigma_{t}^{2}}{k}\left(\Pi_{t}^{j^{*}}-\gamma\right)
\end{aligned}
$$

By the Mean Value Theorem there exists $\eta_{\gamma} \in(\gamma, 1)$ such that

$$
H^{\prime}\left(\Pi^{* j}\right)=H^{\prime}(\gamma)+\left(\Pi^{*^{j}}-\gamma\right) H^{\prime \prime}\left(\eta_{\gamma}\right)
$$

Substituting Equation (15) in Equation (17) the optimal portfolio that maximizes the expected power utility from terminal wealth is written as follows,

$$
\Pi_{t}^{*_{j} j}=\gamma+\frac{H^{\prime}(\gamma)}{\frac{\sigma_{t}^{2}}{k}+\left|H^{\prime \prime}\left(\eta_{\gamma}\right)\right|}
$$

From Equation (18) the holding of excess stock by investors over the continuous optimal $\gamma$ which is strictly due to the presence of jumps is given by,

$$
\Pi_{J}^{* j}=\frac{H^{\prime}(\gamma)}{\frac{\sigma_{t}^{2}}{k}+\left|H^{\prime \prime}\left(\eta_{\gamma}\right)\right|},
$$

The holding of excess stock by investors over the continuous optimal $\gamma$ can be negative or positive depending on $H^{\prime \prime}\left(\eta_{\gamma}\right)$ that is determined numerically.

\subsection{Asymptotic Utilities of Investors}

Let $U_{T}^{j}(x)$ be the maximum expected power utility of the uninformed investor resulting from an optimal portfolio $\Pi_{t}^{j^{*}}$. The risk-adjusted stock's Sharpe ratio for the investor is $\theta_{\alpha}^{j}=\frac{\mu^{j}}{\sigma}=\frac{\mu+v^{j} \sigma}{\sigma}=\frac{\mu^{j}}{\sigma}+v^{j}$ where $\theta_{\alpha}^{j}=\frac{\theta_{t}}{1-\alpha}$ and $r=0$.

From [7] we have the following result.

Theorem 5.3. ([7] Theorem 2.1) Let $x>0$ be the initial wealth of the investor then:

1) The optimal portfolio and maximum expected logarithmic utility from terminal wealth for the investors in a purely continuous market are given by

$$
\Pi_{c}^{j^{*}}=\frac{\theta^{j}}{\sigma}=\frac{\mu^{j}}{\sigma}+\frac{v^{j}}{\sigma^{2}}
$$

and

$$
U_{T, c}^{j}(x)=\log x+\frac{1}{2} E \int_{0}^{T}\left(\theta^{j}\right)^{2} \mathrm{~d} t=\log x+\frac{1}{2} \int_{0}^{T}\left(\frac{\mu_{t}^{j}}{\sigma_{t}}\right)^{2} \mathrm{~d} s+\frac{1}{2} \int_{0}^{T} E\left(v_{t}^{j}\right)^{2} \mathrm{~d} s
$$


2) As $T \rightarrow \infty$ the asymptotic maximum expected utility is:

$$
U_{\infty}^{j}=\log x+\frac{1}{2} \int_{0}^{T}\left(\frac{\mu_{t}^{u}}{\sigma_{t}}\right)^{2} \mathrm{~d} s+\frac{\lambda}{4}(1-p)\left(1+(-1)^{j+1} p\right) T
$$

3) The excess asymptotic maximum expected utility of the informed investor is: $U_{\infty}^{1}-U_{\infty}^{0}=\frac{\lambda}{2}(1-p) p T$.

Now we have maximum expected power utility and asymptotic maximum expected power utility as a result for the jump case which contains the result of [7] when there is no jump and the utility is logarithmic.

\section{Theorem 5.4.}

1) As $T \rightarrow \infty$ the maximum expected power utility from the terminal wealth for investors is given by $U_{\infty}^{j}(x)=\mathrm{e}^{\alpha \log x+\frac{\alpha}{2(1-\alpha)} E \int_{0}^{T}\left(\frac{\mu_{t}+v_{t}^{u} \sigma_{t}}{\sigma}\right)^{2} \mathrm{ds}+\frac{\alpha}{2(1-\alpha)} E \int_{0}^{T} g^{j}\left(\Pi_{t}^{j *}\right) \mathrm{d} s}$.

2) As $T \rightarrow \infty$, the asymptotic maximum expected power utility from the terminal wealth for investors is given by

$$
U_{\infty}^{j}(x)=\mathrm{e}^{\alpha \log x+\frac{\alpha}{2(1-\alpha)} \int_{0}^{t}\left(\frac{\mu_{t}}{\sigma}\right)^{2} \mathrm{ds}+\frac{\lambda}{2}(1-p)\left(1+(-1)^{j+1} p\right) T+\frac{\alpha}{2(1-\alpha)} T \Pi^{*} j}
$$

3) The relative asymptotic maximum expected basis power utility for the informed investor is: $\frac{U_{\infty}^{1}(x)}{U_{\infty}^{0}(x)}=\mathrm{e}^{\frac{\alpha}{2(1-\alpha)} T \lambda(1-p) p+\frac{\alpha}{2(1-\alpha)} T\left(\psi_{\Pi^{* 1}}-\psi_{\Pi^{*}}\right)}$.

\subsection{Utilities under Quadratic Approximation of $\boldsymbol{H}(\gamma)$ and Optimal Portfolios}

To approximate $H(\gamma)$ let as use Taylor expansion and drive some useful formulas. Define $H(\gamma)=\int_{R} \log \left(1+\gamma\left(\mathrm{e}^{z}-1\right)\right) v(\mathrm{~d} z)$ for $\gamma \in[0,1]$ and assume $\int_{R}\left(\mathrm{e}^{z}-1\right)^{n} v(\mathrm{~d} z)<\infty$, there exists $n \geq 0$ which ensures that $H^{(n)}(\gamma)$, the $n^{\text {th }}$ derivative of $H(\gamma)$ exists on $[0,1]$.

The following definition is useful which is connected to the Levy measure that will be used for the computation of approximation.

Definition 5.5. Let $i \in\{1,2, \cdots, n\}$. Define the $i^{\text {th }}$ instantaneous centralized moments of returns for the stock dynamics (2) by:

$$
N(i)=\int_{R}\left(\mathrm{e}^{z}-1\right)^{i} v(\mathrm{~d} z)
$$

$N_{i}$ is well defined because $\int_{R}\left(\mathrm{e}^{z}-1\right)^{n} v(\mathrm{~d} z)<\infty$ and

$$
H^{(i)}(0)=(-1)^{i-1}(i-1) ! \int_{R}\left(\mathrm{e}^{z}-1\right)^{i} v(\mathrm{~d} z)=H^{(i)}(0)=(-1)^{i-1}(i-1) ! N(i)
$$

By defining,

$$
A(1)=\frac{-N(2)}{2\left(\frac{\sigma_{t}^{2}}{k}+N(2)\right)}, A(2)=\frac{N(1) \sigma_{t}}{2\left(\frac{\sigma_{t}^{2}}{k}+N(2)\right)} \text { and } A_{3}=\frac{k N^{2}(1)}{2\left(\frac{\sigma_{t}^{2}}{k}+N(2)\right)}
$$

Then, 


$$
G\left(\theta_{\alpha}^{2}\right)=A(1) \theta_{\alpha}^{2}+A(2) \theta_{\alpha}+A(3) \triangleq G\left(\theta_{\alpha}^{2}\right)=G\left(\theta_{\alpha}^{2}: \sigma, N(1), N(2)\right)
$$

So now we have the following from the quadratic approximation as a result.

\section{Theorem 5.5.}

1) The optimal portfolio for each investor resulting from the quadratic approximation of $H$, where $H(\gamma)$ is defined on $[0,1]$ is given by,

$$
\pi^{*}=\gamma+\frac{N(1)-N(2) \gamma}{\frac{\sigma^{2}}{k}+N(2)}
$$

2) The jump component of the maximum expected power utility for investors resulting from the quadratic approximation of $H$, where $H(\gamma)$ is defined on $[0,1]$ and $\theta_{\alpha}^{u}$ is the risk adjusted Sharpe ratio, is

$$
U_{T, J} \approx \mathrm{e}^{\frac{\alpha}{2(1-\alpha)} E \int_{0}^{T} G\left(\left(\theta_{\alpha}^{j}\right)_{t}: \sigma_{t}, N(1), N(2)\right) \mathrm{d} t}
$$

3) The maximum expected power utility from the terminal wealth for the investor resulting from the quadratic approximation of $H$, where $H(\gamma)$ is defined on $[0,1]$ and $\theta_{\alpha}$ is the risk adjusted Sharpe ratio, is

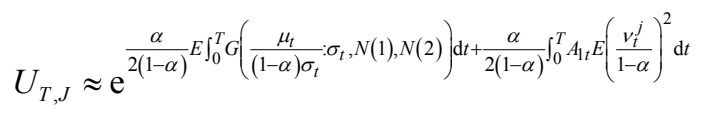

From theorem (5.1) the optimal utility due to the jump for the investor is given by:

$$
\begin{aligned}
U_{T, J} & \triangleq \mathrm{e}^{\frac{\alpha}{2(1-\alpha)} E \int_{0}^{T} g^{j}\left(\Pi_{t}^{j^{*}}\right) \mathrm{d} t} \approx \mathrm{e}^{\frac{\alpha}{2(1-\alpha)} E \int_{0}^{T} G\left(\left(\theta_{\alpha}^{j}\right)_{t} \sigma_{t}, N(1), N(2)\right) d t} \\
& =\mathrm{e}^{\frac{\alpha}{2(1-\alpha)} E \int_{0}^{T}\left(A_{1 t}\left(\theta_{\alpha}^{j}\right)_{t}^{2}+A_{2 t}\left(\theta_{\alpha}^{j}\right)_{t}+A_{3 t}\right) \mathrm{d} t}=\mathrm{e}^{\frac{\alpha}{2(1-\alpha)} \int_{0}^{T}\left(A_{1 t} E\left(\theta_{\alpha}^{u}\right)_{t}^{2}+A_{2 t} E\left(\theta_{\alpha}^{j}\right)_{t}+A_{3 t}\right) \mathrm{d} t}
\end{aligned}
$$

Since $\theta_{\alpha}^{j}=\frac{\theta_{t}}{1-\alpha}=\frac{\mu_{t}^{j}}{(1-\alpha) \sigma_{t}}=\frac{\mu_{t}+v_{t}^{j} \sigma_{t}}{(1-\alpha) \sigma_{t}}=\frac{\mu_{t}}{(1-\alpha) \sigma_{t}}+\frac{v_{t}^{j}}{1-\alpha}$, from lemma $E\left(v_{t}^{j}\right)=0$ then $E\left(\theta_{\alpha}^{j}\right)=\frac{\mu_{t}}{1-\alpha}$.

Hence

$$
\begin{aligned}
E\left(\theta_{\alpha}^{j}\right)^{2} & =\left(\frac{\mu_{t}}{(1-\alpha) \sigma_{t}}\right)^{2}+2 \frac{\mu_{t}}{(1-\alpha) \sigma_{t}} E\left(v_{t}^{j}\right)+E\left(\frac{v_{t}^{j}}{1-\alpha}\right)^{2} \\
& =\left(\frac{\mu_{t}}{(1-\alpha) \sigma_{t}}\right)^{2}+E\left(\frac{v_{t}^{j}}{1-\alpha}\right)^{2} .
\end{aligned}
$$

By substituting Equation (24) in (23)

$$
\begin{aligned}
U_{T, J} & =\mathrm{e}^{\frac{\alpha}{2(1-\alpha)} \int_{0}^{T}\left(A_{1 t}\left(\left(\frac{\mu_{t}}{(1-\alpha) \sigma_{t}}\right)^{2}+E\left(\frac{v_{t}^{u}}{1-\alpha}\right)^{2}\right)+A_{2 t}\left(\frac{\mu_{t}}{1-\alpha}\right)+A_{3 t}\right) \mathrm{d} t} \\
& =\mathrm{e}^{\frac{\alpha}{2(1-\alpha)} \int_{0}^{T}\left(A_{1 t}\left(\frac{\mu_{t}}{(1-\alpha) \sigma_{t}}\right)^{2}+A_{2 t}\left(\frac{\mu_{t}}{1-\alpha}\right)+A_{3 t}\right) \mathrm{d} t+\frac{\alpha}{2(1-\alpha)} \int_{0}^{T} A_{1 t} E\left(\frac{v_{t}^{j}}{1-\alpha}\right)^{2} \mathrm{~d} t} \\
& =\mathrm{e}^{\frac{\alpha}{2(1-\alpha)} E \int_{0}^{T} G\left(\frac{\mu_{t}}{(1-\alpha) \sigma_{t}}: \sigma_{t}, N(1), N(2)\right) \mathrm{d} t+\frac{\alpha}{2(1-\alpha)} \int_{0}^{T} A_{1 t} E\left(\frac{v_{t}^{j}}{1-\alpha}\right)^{2} \mathrm{~d} t} .
\end{aligned}
$$


The following result is asymptotic power optimal utilities due to jump for each investor.

Theorem 5.6.

1) Let $x>0$ be the initial wealth of the investor. By assuming $\int_{R}\left(\mathrm{e}^{z}-1\right)^{n} v(\mathrm{~d} z)<\infty$ and $H(\gamma)$ is defined on $[0,1]$ then the asymptotic optimal power utility for the investor due to jump, as $T \rightarrow \infty$ is given by:

$$
U_{\infty, J}=\mathrm{e}^{\frac{\alpha}{2(1-\alpha)} E \int_{0}^{T} G\left(\frac{\mu_{t}}{(1-\alpha) \sigma_{t}}: \sigma_{t}, N(1), N(2)\right) \mathrm{dt}+\frac{\alpha}{2(1-\alpha)^{3}}(1-p)\left(1+(-1)^{j+1} p\right) \lambda T A_{1 \infty}}
$$

where $A_{1 \infty}=\frac{-\alpha N(2)}{\alpha N(2)+2(1-\alpha) \sigma^{2}}, \quad \sigma=\lim _{t \rightarrow \infty} \sigma_{t}$.

2) Let $x>0$ be the initial wealth of the investor.By assuming $\int_{R}\left(\mathrm{e}^{z}-1\right)^{n} v(\mathrm{~d} z)<\infty$ and $H(\gamma)$ is defined on $[0,1]$ then the excess asymptotic optimal utility of the informed investor over the uninformed, due to jump,as $T \rightarrow \infty$ is given by: $U_{\infty, J}^{1}-U_{\infty, J}^{0}=\lambda A_{1 \infty} p(1-p) T$

By lemma (3.4) as $t \rightarrow \infty \quad E\left(v_{t}^{u}\right)^{2}=\frac{\lambda}{2}(1-p)\left(1+(-1)^{j+1} p\right) T \quad$ and by theorem (5.4) we have,

$$
\begin{aligned}
U_{T, J} & \approx \mathrm{e}^{\frac{\alpha}{2(1-\alpha)} E \int_{0}^{T} G\left(\frac{\mu_{t}}{(1-\alpha) \sigma_{t}}: \sigma_{t}, N(1), N(2)\right) \mathrm{d} t+\frac{\alpha}{2(1-\alpha)} \int_{0}^{T} A_{1 t} E\left(\frac{v_{t}^{u}}{1-\alpha}\right)^{2} \mathrm{~d} t} \\
= & \mathrm{e}^{\frac{\alpha}{2(1-\alpha)} E \int_{0}^{T} G\left(\frac{\mu_{t}}{(1-\alpha) \sigma_{t}}: \sigma_{t}, N(1), N(2)\right) \mathrm{d} t+T \frac{\alpha}{2(1-\alpha)} \lim _{t \rightarrow \infty} A_{1 t} E\left(\frac{v_{t}^{j}}{1-\alpha}\right)^{2}} \\
= & \mathrm{e}^{\frac{\alpha}{2(1-\alpha)} E \int_{0}^{T} G\left(\frac{\mu_{t}}{(1-\alpha) \sigma_{t}}: \sigma_{t}, N(1), N(2)\right) \mathrm{d} t+\frac{\alpha}{2(1-\alpha)(1-\alpha)^{2}}(1-p)(1-p) \lambda T A_{1 \infty}}
\end{aligned}
$$

where $A_{1 \infty}=\lim _{t \rightarrow T} A_{1 t}=\frac{-\alpha N(2)}{\alpha N(2)+2(1-\alpha) \sigma_{t}^{2}}, \quad \sigma=\lim _{t \rightarrow \infty} \sigma_{t}$.

Theorem 5.7. The total asymptotic excess optimal utility of the informed investor over the uninformed, as $T \rightarrow \infty$ is given by.

$$
U_{\infty}^{1}-U_{\infty}^{0}=\frac{\lambda^{*}}{2} p(1-p) T,
$$

where $p \in[0,1]$ and $x>0$ is the intial wealth of investors $\sigma=\lim _{t \rightarrow \infty} \sigma_{t}$.

Theorem 5.8. As $T \rightarrow \infty$ the maximum expected power utility from the terminal wealth for investors with initial wealth $x>0$ and $p \in[0,1]$ is given by:

$$
U_{\infty}^{j}(x)=\mathrm{e}^{\alpha \log x+\frac{\alpha}{2(1-\alpha)} \mathrm{t}_{0}^{t}\left(\frac{\mu_{t}}{\sigma_{t}}\right)^{2} \mathrm{ds}+\frac{\alpha}{2(1-\alpha)} \int_{0}^{t} G\left(\frac{\mu_{t}}{(1-\alpha) \sigma_{t}}\right)^{2} \mathrm{ds}+\frac{\alpha}{2(1-\alpha)^{3}}(1-p)\left(1+(-1)^{j+1} p\right) 2 T A_{1 \infty}}
$$

Using theorem (5.1) and theorem (5.6) it is the direct substitution. From theorem (5.4), since $g^{j}\left(\Pi^{j}\right)$ is continuous, then by mean value theorem,

$$
\lim _{T \rightarrow \infty} \frac{1}{T} E \int_{0}^{t} g^{j}\left(\Pi_{t}^{j^{*}}\right) \mathrm{d} t=\lim _{t \rightarrow \infty} E g^{j}\left(\Pi_{t}^{j^{*}}\right)=\psi_{\Pi^{* j}}
$$

Thus, $E \int_{0}^{t} g^{j}\left(\Pi_{t}^{j^{*}}\right) \mathrm{d} t=T \psi_{\Pi^{* j} j}$ and $\int_{0}^{t} E\left[v_{t}^{j}\right] \rightarrow \frac{\lambda}{2}(1-p)\left(1+(-1)^{j+1} p\right) T$ as $T \rightarrow \infty$ 
So the explicit formula for $T \psi_{\Pi^{*}{ }^{*}}$ by solving Equation (20) and Equation (26) is written as follows.

$$
\begin{aligned}
& \frac{1}{T} \lim _{t \rightarrow \infty} \operatorname{Eg}^{u}\left(\Pi_{t}^{* j}\right)=\psi_{\Pi^{* j}} \\
& =\frac{1}{T} \int_{0}^{t} G\left(\frac{\mu_{t}}{(1-\alpha) \sigma_{t}}\right)^{2} \mathrm{~d} s+\frac{1}{(1-\alpha)^{2}}(1-p)^{2} \lambda A_{1 \infty}-\frac{(1-\alpha) \lambda}{\alpha}(1-p)^{2}
\end{aligned}
$$

\section{Conclusions}

We derived the optimal portfolios, maximum expected power utilities and asymptotic utilities for each investor from the terminal wealth when there are inefficiencies in the market. The traded stock or risky asset price is modeled as a Levy jump process by specifying the asset price process in the large filtration of informed investor, and then obtaining its dynamics for uninformed investor using the Hitsuda representation of Gaussian processes assuming there are two distinct classes of rational investors in a financial market.

Using quadratic approximation of the portfolios, we show that extra assets are detained by an investor if and only if the ratio of the first and second instantaneous Central moments of return is larger than the continuous optimal portfolio of that investor. We also show that jumps minimize the excess long run utility for the informed investor relative to that of the uninformed investor, which implies that jump risk may be good for market efficiency as an unintended reducer of information asymmetry.

\section{Conflict of Interests}

The authors declare that there is no conflict of interests regarding the publication of this paper.

\section{Acknowledgements}

We are grateful to the authors for their numerous and valuable contributions to this work.

\section{References}

[1] LeRoy, S.F. and LaCivita, C.J. (1981) Risk Aversion and the Dispersion of Asset Prices. The Journal of Business, 54, 535-547. https://doi.org/10.1086/296145b

[2] Shiller, R. (1981) Do Stock Prices Move Too Much to Be Justified by Subsequent Changes in Dividends? American Economic Review, 71, 421-436.

[3] Kelly, B. and Ljungqvist, A. (2012) Testing Asymmetric-Information Asset Pricing Models. The Review of Financial Studies, 25, 1366-1413. https://doi.org/10.1093/rfs/hhr134

[4] Vayanos, D. and Wang, J. (2012) Theories of Liquidity. Foundations and Trends $R$ in Finance, 6, 221-317.

[5] Mendel, B. and Shleifer, A. (2012) Chasing Noise. Journal of Financial Economics, 104, 303-320. https://doi.org/10.1016/j.jfineco.2011.02.018 
[6] Summers, L.H. (1986) Does the Stock Market Rationally Reflect Fundamental Values? The Journal of Finance, 41, 591-601. https://doi.org/10.1111/j.1540-6261.1986.tb04519.x

[7] Guasoni, P. (2006) Asymmetric Information in Fads Models. Finance and Stochastics, 10, 159-177. https://doi.org/10.1007/s00780-006-0006-4

[8] Wang, J. (1993) A Model of Intertemporal Asset Prices under Asymmetric Information. Review of Economic Studies, 60, 249-282. https://doi.org/10.2307/2298057

[9] Applebaum, D. (2009) Levy Processes and Stochastic Calculus. Cambridge University Press, Cambridge. https://doi.org/10.1017/CBO9780511809781

[10] Protter, P.E. (2005) Stochastic Differential Equations. In: Stochastic Integration and Differential Equations, Springer, Berlin, Heidelberg, 249-361. https://doi.org/10.1007/978-3-662-10061-5_6

[11] Hitsuda, M. (1968) Representation of Gaussian Processes Equivalent to Wiener Process. Osaka Journal of Mathematics, 5, 299-312. http://projecteuclid.org/euclid.ojm/1200692174

[12] Amendinger, J., Imkeller, P. and Schweizer, M. (1998) Additional Logarithmic Utility of an Insider. Stochastic Processes and Their Applications, 75, 263-286.

http://www.sciencedirect.com/science/article/pii/S0304414998000143 https://doi.org/10.1016/S0304-4149(98)00014-3

[13] Ankirchner, S., Imkeller, P., et al. (2007) Financial Markets with Asymmetric Information: Information Drift, Additional Utility and Entropy. Stochastic Processes and Applications to Mathematical Finance, 1-21.

[14] Liu, J., Longstaff, F.A. and Pan, J. (2003) Dynamic Asset Allocation with Event Risk. The Journal of Finance, 58, 231-259. https://doi.org/10.1111/1540-6261.00523 